\title{
El efecto movilizador de las emociones sobre la participación política online en la segunda vuelta de las elecciones presidenciales en Ecuador 2017
}

\author{
The mobilizing effect of emotions on online political \\ participation in the second round of presidential elections in \\ Ecuador 2017
}

Marcos Zumárraga Espinosa, Cynthia Carofilis Cedeño y

Carlos Reyes Valenzuela

Recepción: 30 de junio de 2017

Aceptación: 23 de agosto de 2017

\section{Resumen}

Las recientes elecciones presidenciales en Ecuador durante la campaña de los candidatos Lenín Moreno y Guillermo Lasso, suscitaron un uso creciente de las redes sociales. El objetivo del presente estudio es analizar cómo las emociones hacia los dos candidatos promueven la participación política en redes sociales y online, en una muestra de 1136 participantes de la ciudad de Quito. Los resultados evidencian que las emociones sean positivas o negativas, cumplen un rol movilizador sobre la ciudadanía en términos de activismo político vía internet. Asimismo, el candidato Moreno genera estímulos emocionales con un efecto movilizador sobre el electorado, efecto que no se verifica en el caso del candidato Lasso. Se discuten las implicaciones de estos resultados en un contexto de creciente debate en Ecuador sobre la necesidad de establecer mecanismos de control a su uso.

Palabras clave: participación política online, redes sociales, emociones, elecciones presidenciales, Ecuador.

\section{Abstract}

The recent presidential elections in Ecuador during the campaign of the candidates Lenin Moreno and Guillermo Lasso, raised an increasing use of the social networks. The objective of the present study is to analyze how the emotions towards both candidates promote the political participation in social and online networks, in a sample of 1136 participants of Quito city. The results show that either positive or negative emotions play a mobilizing role on citizenship in terms of political activism through internet. Likewise, the candidate Moreno generates emotional stimuli with a mobilizing effect on the electorate, the same effect can't be verified in the case of candidate Lasso. The implications of these results are discussed in a context of growing debate in Ecuador about the need to establish control mechanisms to its use.

Key words: online political participation, social networks, emotions, presidential elections, Ecuador. 


\section{Elecciones presidenciales en Ecuador}

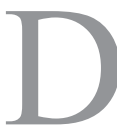

espués de diez años de gobierno bajo el mandato presidencial de Rafael Correa Delgado, en los cuales el movimiento Alianza PAIS dispuso de una mayoría absoluta de asambleístas, las recientes elecciones presidenciales llevadas a cabo en Ecuador representaron un escenario donde se puso en juego tanto la legitimidad del modelo del "socialismo del siglo XXI", como la posibilidad de un futuro más democrático para el país. Estas elecciones se producen en medio de un contexto político regional y nacional de alta inestabilidad, tales como el escándalo mediático de Odebrecht, el estallido de la crisis política en Venezuela o la caída del gobierno de Dilma Rousseff en Brasil, la reelección presentaba diversas amenazas para la continuidad del modelo político correísta. En este entorno, gran parte de la discusión se entabló en las redes sociales por medio de iracundos ataques entre el sector oficialista y el de oposición, en una creciente ola de actividad en la que los memes, mensajes, audios y rumores de desprestigio, opacaron las propuestas de dos modelos políticos opuestos.

Los resultados de la primera vuelta electoral celebrada el 19 de febrero confirmaron una segunda vuelta entre los candidatos Lenín Moreno (por el partido Alianza PAIS) y Guillermo Lasso (por el movimiento CREO). Moreno, ex vicepresidente en el primer ciclo de Rafael Correa (2008-2012) personificaba la continuidad de un modelo que apuesta fuertemente por la inversión estatal y los bienes públicos, el neo-extractivismo y la prolongación del modelo económico y político actual. Por su parte, Lasso, representante del sector empresarial guayaquileño, prometía recuperar las libertades perdidas, la generación de un millón de empleos y la abolición de algunos impuestos.

Como parte de la campaña, ambos candidatos utilizaron profusamente las redes sociales como "tarimas electorales": los debates en Facebook, Twitter y Whatsapp se convirtieron en espacios de difusión de informaciones referentes a los escándalos antes mencionados, con fotografías, documentos y testimonios que cuestionaban la legitimidad de los resultados obtenidos en primera vuelta y hacían un llamado a la vigilancia en las urnas. El 2 de abril de 2017, día de celebración de la segunda vuelta, con anterioridad al anuncio 
oficial del Consejo Nacional Electoral, algunos medios proclamaron ganador a Guillermo Lasso y, posteriormente, otros presentaron a Lenín Moreno celebrando la victoria. La página oficial del CNE colapsó durante 20 minutos, cuestión que terminó de encender la desconfianza en los resultados, al calor de los rumores de un posible "apagón informático".

Convocados por Lasso y su equipo, sus partidarios salieron a las calles ese mismo día, iniciando una serie de protestas que se disolvieron quince días después con el reconteo oficial de los votos. La impugnación de resultados fue petición de ambos partidos, en la cual el movimiento CREO y Alianza PAIS solicitan el reconteo de 4243 actas y 714, respectivamente. En definitiva, el 18 de abril en un acto público, en presencia de miembros de la sociedad civil, academia y organizaciones políticas, los resultados finales otorgaron una décima más para Lenín Moreno $(51,16 \%)$ y una menos para Guillermo Lasso (48,84\%). En este punto, se observa que el uso de redes sociales se configura como un mecanismo clave en la difusión y debate de información de diversos temas políticos y la promoción de la participación de la ciudadanía ecuatoriana que, en ese periodo, se caracterizó porque un amplio grupo mostraba indecisión respecto a su elección de voto.

\section{Datos del uso de redes sociales en Ecuador}

En Ecuador hasta julio del 2012, los usuarios de Internet constituyendo un total de 7.320.206 que representa el $50,5 \%$ de la población, según cifras estadísticas de la Corporación Nacional de Telecomunicaciones (Punín, Martínez, \& Rencoret, 2014). A nivel nacional, el 36\% de los hogares tienen acceso a internet, 13,5 puntos más que hace cinco años. En el área urbana, el crecimiento es de 13,2 puntos, mientras que en la rural de 11,6 puntos. En 2016, la tenencia de teléfonos inteligentes (smartphone) creció entre 2015 al 2016 del 37,7\% al 52,9\%, en relación con la población que posee un celular activado. Al finalizar el año 2016, los usuarios de redes sociales llegaban a 4.224.984 ciudadanos (Instituto Nacional de Estadísticas y Censos, 2016).

Se ha considerado que estas posibilidades de conectividad están ligadas a oportunidades conducentes a nuevas formas de participación. De 
acuerdo con el Informe del Latinobarómetro 2015, el Ecuador presentó un cambio desde el denominado "hiper-presidencialismo" a la "hiperparticipación", caracterizada por una dinámica ciudadana de salir a la calle a defender derechos, acciones impulsadas a través de las redes sociales. En 2015, el 41,7\% de los encuestados utilizaba Internet para buscar información (Corporación Latinobarómetro, 2016) lo que revelaba el ingente uso de las redes sociales como una fuente importante de búsqueda que compite con los medios tradicionales. Además, en los últimos años, las redes sociales se han configurado como un espacio de intercambio no sólo progresivamente mayor de las opiniones políticas, sino de denuncia, interacción y comunicación con las instituciones públicas. Esto promueve la apertura de diversos canales por parte de figuras políticas e instituciones, especialmente públicas, que utilizan las redes sociales como plataforma para el debate político, aspecto que detona con el aprovechamiento de estas plataformas digitales por parte de los candidatos presidenciales en la reciente campaña electoral (Carvajal, 2017).

Este interés en utilizar las redes sociales durante la campaña presidencial tiende a fundamentarse en el creciente uso político que la ciudadanía hace de las mismas: desde un aumento en el número de personas que siguen en Facebook, audiencias en Youtube respecto a información sobre los candidatos o la creación de grupos de Whatsapp como medios de difusión de campañas. Al finalizar la primera vuelta y confirmando la presencia de Moreno y Lasso como candidatos, el uso de redes sociales expresó el clima de polémica y discusión viral de los resultados, como por ejemplo, los hashtags ${ }^{1}$ \#Manabíserespeta, \#Fraude y \#HaySegundaVuelta que revelaron no solo el acceso a la información mediante estas redes, sino también convocatorias a tomarse las calles. En resumen, el uso de las redes sociales durante las elecciones presidenciales resulta indiscutible, aunque con matices diferenciados dependiendo de las posturas políticas.

Esto se evidencia en que para algunos, dado el contexto limitado de participación ciudadana durante el periodo de Rafael Correa, las redes

1. Nota del Editor: HASHTAG-Es una etiqueta formada por una o varias palabras precedidas por el signo numeral (\#) que permite la rápida búsqueda o posicionamiento de un tema. Es utilizada en diversas redes sociales como: Twitter, Telegram, Facebook, Instagram, Google+, entre otras. 
sociales representan un potencial democratizador o, por el contrario, un punto de origen para el fenómeno de troll centers $^{2}$, que promueve múltiple información, principalmente negativa, a través dichas redes, sea a favor del gobierno (Rojas, 2017) o en su contra. Por tanto, dado lo nuevo de este uso, el Código de la Democracia no contempla la censura para las redes sociales (“Campaña electoral se anticipa en las redes," 2016) aspecto que sí se cuestiona posteriormente, a partir del envío a la Asamblea de un proyecto de ley para la regulación de las redes sociales. Basada en los artículos del Código Integral Penal que definen los discursos y delitos de odio, dicho proyecto pretende sancionar la difamación en los espacios virtuales, a raíz de los eventos ocurridos a partir de la primera vuelta electoral.

La iniciativa legal de regulación de las redes sociales plantea el debate respecto de los mecanismos de control versus la libertad de expresión. A pesar de este foco, uno de los aspectos que ha llamado la atención y que está condicionando el análisis sobre dicho debate, se refiere al papel de las emociones en el uso de las redes sociales y de qué modo dificultan o posibilitan la participación política. Complementario a esto, está la consideración sobre si la participación online o en redes sociales favorece la participación política. Estos aspectos serán tratados a continuación.

\section{Emociones, participación online y uso de redes sociales}

Las emociones se originan en los intercambios sociales e inciden en la evaluación que las personas realizan de sus contextos y la motivación hacia la acción (Lazarus \& Folkman, 1986) ya que pone de manifiesto un conjunto de significados y creencias compartidas socialmente (Otero, 2006). Las emociones movilizan e impulsan hacia las acciones, en la cual pueden distinguirse dos niveles: uno individual, ya que las emociones favorecen un impulso inicial y luego uno colectivo, estructurando un aspecto esencial en la identidad y cohesión. Esto último configuraría las condiciones en las cuales las emociones promoverían la movilización social (Castillo, 2012) y, por lo

2. Nota del Editor: TROLL CENTER-Utilizado en redes sociales hace referencia a las personas o mecanismos creados con cuentas reales o falsas para realizar ataques sistemáticos ya sea provocando, criticando, o insultando marcas o usuarios de estas redes. 
tanto, la participación política.

Durante mucho tiempo las emociones se consideraron como las antípodas de la conducta política, principalmente porque los teóricos sociales siguieron las dicotomías que oponen la razón a la emoción y el cuerpo a la mente. Progresivamente, las ciencias políticas y la psicología han incorporado en sus análisis el papel central de las emociones en la política, bajo el supuesto clave de que las emociones no son lo opuesto a lo racional, sino más bien se constituyen en catalizadoras del aprendizaje político (Marcus \& MacKuen, 1993).

El modelo de inteligencia afectiva (Marcus, Neuman, \& Mackuenn, 2007) propone un proceso dual en el surgimiento de las emociones, en la cual nos proporciona una guía para interpretar nuestras sensaciones y servirían como facilitadoras para el procesamiento cognitivo. Este modelo funciona a través de dos sistemas: disposicional, que maneja la evaluación de las conductas aprendidas y de lo conocido, en la cual son indispensables las emociones positivas y negativas que capturan los gustos y disgustos relativos al estímulo político (por ejemplo, la preferencia por un candidato). En tanto un segundo sistema, vigilancia, evalúa la entrada del flujo de sensaciones, cuando se encuentra con algo desconocido o inesperado, responde con ansiedad, lo que, en el ámbito político, se relacionaría con una disminución en la orientación a la acción política (por ejemplo, inhibiendo la participación política).

De acuerdo con este modelo, para los estímulos políticos, la ansiedad inhabilita la actividad habitual de los votantes, requiriendo que las personas se detengan, presten atención y consigan información nueva y precisa. En este sentido, las personas cuando están ansiosas están más atentas a los estímulos políticos y pueden reconsiderar sus posiciones ideológicas. Por el contrario, cuando en el electorado se presentan emociones positivas, no se detienen a dudar y se involucran de lleno en las actividades de campaña. De ahí que para este modelo, se entiende a las emociones como heurísticas que configuran un atajo para adoptar sus juicios y decisiones políticas (Marcus et al., 2007). A pesar de esto último, se ha considerado que los efectos de mensajes emocionales en el ámbito político variarán acorde al contexto en el que se reciban y lo que define su impacto depende de estímulos (no solo emocionales) que permitan activar un marco específico para la acción política (Castillo, 2012). 
En Internet, las emociones son desplegadas y moduladas, lo que posibilita configurar la identidad de la persona (Serrano-Puche, 2016). Dicho de otro modo, en la Red las emociones son fundamentales para los intercambios y comunicación que se presenta, puesto que Internet presenta diferentes entornos en los cuales las emociones surgen. Por ejemplo, se ha encontrado que las emociones positivas que se expresan online presentan un carácter similar a las interacciones sociales y, en cambio, las emociones negativas intensas se expresan más claramente en Internet (Serrano, 2016). Respecto a los mensajes y contenidos políticos en Internet, es posible asociarlos a la noción de "flujo emocional", en el cual las noticias e información política se repite en la Red continuamente lo que deriva en el incremento de un input (ingreso) más emocional que cognitivo (Papacharissi \& Oliveira, 2012).

En relación a la imagen de los candidatos y de las emociones que pueden generar, de acuerdo a Zamora y Losada (2011), la construcción de la imagen se define en relación a otras características que posibilitan la formación de una percepción entre el electorado, en la cual influye la situación política o económica del contexto. Sin embargo, es posible pensar que en un entorno de Red, la imagen de un candidato es esencialmente de tipo emocional, en la cual factores externos como la situación política, económica u otras, contribuirán en la construcción de una imagen positiva o negativa, dependiendo de cómo se realiza la expresión emocional online de la ciudadanía.

En otro aspecto, es necesario considerar qué es participación online, que puede ser identificada como una acción cuyo escenario es la red. Aunque no hay consenso en la definición de participación online, algunos investigadores hacen la distinción entre la discusión política y la acción política, dado que la expresión política es conceptualmente distinta de la participación política, de la misma manera que las discusiones son distintas de las acciones (Gil de Zúñiga, Veenstra, Vraga, \& Shah, 2010). De acuerdo a esta definición, la noción de participación política online plantea ciertos argumentos a tener en cuenta, puesto que se han identificado una diversidad de actividades y no hay claridad si dichas actividades propician una participación. En este sentido, consideramos útil seguir la distinción entre uso activo y pasivo de las redes, ya que se relacionan de manera diferencial con la participación offline. 
Para Gibson, Lusoli y Ward (2005) la participación online es multidimensional, es decir, las actividades en internet se agrupan dando lugar a un tipo de participación activa y otra pasiva. Las autoras a partir de un modelo contextualizado de participación distinguen entre cuatro dominios: a) E-party, que involucra varias formas de actividades voluntarias para un partido como donar dinero en línea, b) E-targeting, que se relaciona con el establecimiento de contactos en la web, c) E-expresive, que comprende diferentes modos de discusión política y, d) E-news, que implica obtener información a través de sitios web de partidos y medios (R. Gibson \& Cantijoch, 2013, p. 711). La participación online activa incluiría las dos primeras y la participación pasiva, las dos últimas. De acuerdo con esto, en el entorno online, las formas expresivas e informativas de participación tendrían mayor influencia dada la facilidad de acceso a la información y la posibilidad de alcanzar a una mayor audiencia, en comparación con formas clásicas de participación como leer un periódico o discutir entre conocidos sobre política.

Otra forma en la que se ha entendido la participación política online se refiere a la relación que se establece respecto a la participación política offline, esto es, la participación que ocurre en un escenario fuera de la red. Al respecto, se plantea que las actividades de participación offline implican un mayor costo para las personas, en tanto que las online son de bajo costo (Vaccari et al., 2015). De este modo, la participación política online es más fácil de acceder, implica menos recursos económicos y técnicos o posibilita la interacción y creación de contenidos (Vaccari et al., 2015). Además, permite la participación de personas y grupos que no disponen de tales recursos o que constituyen minorías (Bekafigo \& Mcbride, 2013; R. K. Gibson et al., 2005). Este argumento concatena con las críticas a la participación online porque no necesariamente conduce a otras formas de participación offline, puesto que estas actividades crean en la gente la ilusión de ser políticamente activa (Shulman, 2009) o conducen más a una participación ciudadana que política (Zhang, Johnson, Trent Seltzer, \& Bichard, 2009).

En contra de esta última línea, hay estudios que establecen que ser usuario de internet resulta un predictor para la participación tanto online como offline (Yang \& Dehart, 2016), especialmente en jóvenes (EspinarRuiz \& González-Río, 2015). Complementario a esto, hay investigaciones 
que sugieren que el uso de redes sociales propicia la participación offline (De Marco \& Robles Morales, 2012; Vaccari et al., 2015; Valenzuela, Park, $\&$ Kee, 2009). De esta manera, podemos distinguir en la participación política en un entorno online dos tipos: una participación propiamente online que incluye actividades que se realizan en la red. Una segunda, que está caracterizada por la participación política en redes sociales (tales como Facebook, Youtube, Whatsapp). En este punto, interesa conocer el papel diferenciador de las emociones en ambas formas de participación. Para ello, se adopta una estrategia de control de algunas variables que están conceptualmente relacionadas con la participación política. Dichas variables se asocian a características socio-demográficas, recursos de participación, factores digitales, motivaciones, actitudes y movilización externa.

En el caso de recursos se considera el indicador estatus socioeconómico (Verba, Schlozman, Lehman, \& Brady, 1995). El bloque de factores digitales contempla las variables uso de internet y eficacia política de internet (Bosnjak, Galesic, \& Klicek, 2007; Nam, 2012). En el bloque de motivaciones y actitudes se incluyen las siguientes variables: interés en la política, confianza en el gobierno, confianza interpersonal, ideología política, responsabilidad moral frente a la política, opinión del círculo social percibida, importancia de la democracia, eficacia política interna y eficacia política externa (Bosnjak et al., 2007; De Marco \& Robles Morales, 2012; Jiang, 2016; Lehman et al., 2010; Yang \& Dehart, 2016). Finalmente, los indicadores de movilización externa por parte de organizaciones políticas son la pertenencia a grupos políticos y el contacto online con organizaciones o agentes políticos (De Marco \& Robles Morales, 2012; Jiang, 2016; Perea et al., 2010).

\section{Objetivos e hipótesis del estudio}

Los objetivos del estudio son los siguientes: en primer lugar, explorar el papel de las emociones en la participación política online, tanto en su forma general como a través de redes sociales, en la segunda vuelta de las elecciones presidenciales en Ecuador. De este modo se pretende detectar si las emociones tienen un potencial movilizador en el accionar político en un entorno online y, segundo, qué tipo de emociones promueven tal efecto. En 
tercer lugar, se busca conocer las diferencias que plantea para la participación política online general y en redes sociales el efecto de las emociones. Por último, se indaga las posibles diferencias que este efecto movilizador pueda experimentar para los candidatos Moreno y Lasso en el contexto de elección.

Para esto, se han establecido las siguientes hipótesis:

Hipótesis 1. Las emociones promoverán un efecto movilizador en la ciudadanía en el entorno online de participación política.

Hipótesis 2. Las emociones, con independencia de su valencia, originarán dicho efecto movilizador en la participación política en un entorno online.

Hipótesis 3. Las emociones presentarán mayor efecto en la participación política de redes sociales que en la participación online considerada de modo general.

Hipótesis 4. El efecto movilizador de las emociones sobre las formas de participación política online se producirá de modo diferenciado según se trate del candidato presidencial Lenín Moreno o Guillermo Lasso.

\section{Estudio Cuantitativo}

Participantes y procedimiento

La muestra estuvo conformada por 1136 participantes adultos, de nacionalidad ecuatoriana, que viven en el Distrito Metropolitano de Quito (DMQ), cuyas características sociodemográficas se presentan en la Tabla 1. La edad promedio de los participantes fue de 34,3 años. Tanto la edad promedio como la proporción de mujeres se encuentran ligeramente sobrerrepresentadas en la muestra a comparación con los datos nacionales (según estimaciones del INEC-Ecuador para el 2017, portal web ecuadorencifras, el porcentaje de mujeres es de 50,5\% y la edad promedio es de 29,3 años a nivel nacional). No obstante, aunque la muestra empleada es solamente cuasi representativa a nivel nacional, los resultados obtenidos, guardando las reservas necesarias, pueden ofrecer una buena aproximación de la realidad ecuatoriana en el ámbito de la participación política. 
Los datos empleados se recolectaron mediante la aplicación de una encuesta general de opinión y participación política, diseñado por el Grupo de Investigaciones Psicosociales (GIPS) de la Universidad Politécnica Salesiana (UPS-Sede Quito). Las encuestas se aplicaron durante las semanas previas a la segunda vuelta de las elecciones presidenciales y fueron realizadas por estudiantes de segundo nivel de la Carrera de Psicología de la UPS quienes recibieron la capacitación necesaria en técnicas de encuesta. Los participantes formaron parte del estudio de manera voluntaria, una vez informado de los propósitos y las condiciones de confidencialidad.

\section{Tabla 1.}

Características sociodemográficas de los participantes del estudio

\begin{tabular}{|c|c|c|}
\hline & & $\%$ \\
\hline \multirow{2}{*}{ Sexo } & Masculino & 46,9 \\
\hline & Femenino & 53,1 \\
\hline \multirow{6}{*}{ Educación } & Ninguno, no tiene educación formal & 0,7 \\
\hline & Primaria incompleta y terminada & 3,7 \\
\hline & Secundaria incompleta y terminada & 8,2 \\
\hline & Bachillerato incompleto y terminado & 45,2 \\
\hline & Universidad incompleta y terminada & 40,7 \\
\hline & Postgrado (maestría, doctorado) & 1,5 \\
\hline \multirow{8}{*}{ Ingresos Familiares } & Desde 0 hasta 374 USD & 13,8 \\
\hline & Desde 375 hasta 749 USD & 24,7 \\
\hline & Desde 750 hasta 1124 USD & 29,1 \\
\hline & Desde 1125 hasta 1499 USD & 12,1 \\
\hline & Desde 1500 hasta 1874USD & 7,3 \\
\hline & Desde 1875 hasta 2249 USD & 5,6 \\
\hline & Desde 2250 hasta 2624 USD & 3 \\
\hline & 2625 USD o más & 4,6 \\
\hline \multirow{2}{*}{ Situación ocupacional } & Trabaja actualmente & 53,1 \\
\hline & No trabaja actualmente & 46,9 \\
\hline \multirow{5}{*}{ Edad } & De 17 a 28 años & 47,6 \\
\hline & De 29 a 40 años & 17,4 \\
\hline & De 41 a 51 años & 19,7 \\
\hline & De 52 a 64 años & 12,5 \\
\hline & De 65 años en adelante & 2,8 \\
\hline
\end{tabular}

Fuente: Datos recopilados por el Grupo de Investigaciones Psicosociales (GIPS), Universidad Politécnica Salesiana (UPS), 2017.

Elaborado por: los autores. 


\section{Instrumentos}

\section{Variables dependientes}

Participación Política Online. Se construyó un índice aditivo que incluyó 14 actividades políticas realizables vía internet.

Se contemplaron actividades de carácter expresivo como discutir; publicar comentarios; participar en foros o intentar persuadir a otros para que apoyen o se opongan a algún proyecto político, las campañas o los candidatos presidenciales. Asimismo, se incorporaron actividades de consumo y difusión de información política a través de internet como visitar páginas web; ver videos en Youtube; reenviar correos electrónicos; leer noticias o suscribirse a un sitio web para recibir información de carácter político, de los candidatos o las campañas electorales. Finalmente, se consideraron actividades políticas online de mayor alcance como recolectar o contribuir con dinero; firmar o recolectar firmas; ponerse en contacto con algún político o funcionario público o hacer trabajos, utilizando herramientas y aplicaciones de internet para promover a un candidato, partido político o campaña presidencial.

El nivel de participación en cada actividad se midió a través de una escala Likert ${ }^{3}$ de 5 puntos que varían desde: 1 (Nunca lo he hecho y nunca lo haría bajo ninguna circunstancia), 2 (No lo he hecho pero podría hacerlo), 3 (Lo he hecho una o muy pocas veces), 4 (Lo he hecho algunas veces) o 5 (Lo he hecho muchas veces). La consistencia interna de la métrica global resultante es estadísticamente satisfactoria, presentando un coeficiente Alfa de Cronbach $(\alpha)$ de 0,90. Para la construcción de este índice se tomaron como referencia los trabajos de Sheppard (2015), Anduiza y cols. (2010) y Milošević-Đorđević y Žeželj (2016).

\footnotetext{
3. Nota del editor: La escala de Likert es una herramienta de medición que, a diferencia de preguntas dicotómicas con respuesta sí/no, nos permite medir actitudes y conocer el grado de conformidad del encuestado con cualquier afirmación que le propongamos. Resulta especialmente útil emplearla en situaciones en las que queremos que la persona matice su opinión. En este sentido, las categorías de respuesta sirven para capturar la intensidad de los sentimientos del encuestado hacia dicha afirmación.
} 
Participación Política en Redes Sociales: Se midió la participación política mediante las redes sociales de Facebook, Twitter y Whatsapp.

El uso político de cada red social se evaluó a través de ocho conductas de carácter político que pueden tener lugar en dicha plataforma digital. Las actividades contempladas van desde iniciar o unirse a un grupo; escribir, comentar o responder opiniones; compartir contenido; chatear con otros sobre política; hasta recibir contenidos, imágenes, videos y links relacionados con cuestiones políticas, los candidatos o las campañas electorales.

Se calculó a través de una escala Likert de 5 puntos, desde 1 (Nunca) a 5 (Siempre). La consistencia interna de cada subescala presentó un nivel satisfactorio: Facebook $(\alpha=0,90)$, Twitter $(\alpha=0,94)$ y Whatsapp $(\alpha=0,92)$. Finalmente, se sumaron los puntajes de cada subescala en una sola métrica aditiva global que integre las redes sociales de interés, presentando igualmente una consistencia interna satisfactoria $(\alpha=0,95)$. Para la construcción de las subescalas de cada red social se tomaron como referencia los trabajos de Sheppard (2015), Milošević-Đorđević y Žeželj (2016), Anduiza et al. (2010) y Bekafigo \& Mcbride (2013).

\section{Variables Explicativas}

Emociones: Las emociones discretas contempladas en el presente estudio fueron: entusiasmo, desprecio, odio, esperanza, miedo, indignación, amargura, ansiedad, resentimiento, orgullo, preocupación y enfado. (Redlawsk, 2006).

Se preguntó a los encuestados en qué medida sentían cada una de estas emociones con respecto a cada candidato presidencial por separado: Lenín Moreno y Guillermo Lasso. Las opciones de respuesta estuvieron en un rango que va desde 1 (Nada) hasta 5 (Extremadamente). A través de un análisis factorial exploratorio (AFE) se identificaron dos dimensiones subyacentes, en la cual el primer factor incluyó las emociones de desprecio, odio, miedo, indignación, amargura, ansiedad, resentimiento, preocupación y enfado, que se categorizaron como emociones negativas. El segundo factor se formó con las emociones de entusiasmo y esperanza, consideradas como positivas. La emoción 
de orgullo presentó cargas factoriales similares en los dos factores encontrados, razón por la cual fue descartada. La consistencia interna es satisfactoria para emociones positivas $(\alpha=0,80)$ y emociones negativas $(\alpha=0,93)$.

\section{Variables de Control}

Características socio-demográficas: Se realizaron controles para el efecto del sexo y la edad a fin de evitar algún impacto de estas variables sobre las dependientes.

Recursos: La disponibilidad de recursos cognitivos y materiales para superar los costos de participación política en el entorno online se midió a través de la variable estatus socioeconómico, que incluye el nivel de educación e ingreso equivalente. El ingreso equivalente se calculó dividiendo el ingreso familiar reportado por el encuestado por la cantidad de personas que viven en su hogar (Castillo, Miranda \& Madero, 2013). El estatus socioeconómico puede concebirse como la interacción entre educación e ingresos, por lo que se procedió a estandarizar sus respectivos indicadores en puntajes z y obtener su producto, tal cual fue utilizado en estudios anteriores (Morris \& Morris, 2013).

Factores Digitales: El uso de internet se empleó como un indicador de las habilidades digitales con las que cuenta una persona y se evaluó en dos variables: uso de internet y eficacia política de internet. De esta manera, se pretende considerar la brecha digital de segundo nivel (habilidades digitales) que va más allá de disponer acceso a internet de forma habitual (Morris \& Morris, 2013). Esto permite captar la influencia de las diferencias entre quienes tienen mayores y menores habilidades digitales en relación con las formas de participación política online. Primero, el uso de internet se midió a través de un ítem en una escala de frecuencia de 6 puntos que va desde 1 (Casi nunca) a 6 (Siempre). En segundo término, la eficacia política de internet se evaluó mediante un ítem en una escala de 5 puntos que evalúa el grado de confianza que los participantes tienen en internet como un medio útil para influir en asuntos políticos. Las opciones de respuesta se sitúan en un rango que va desde 1 (Nada) a 5 (Mucho). 
Motivaciones y Actitudes: Fueron evaluadas a través de nueve escalas: en primer lugar, el interés en la política se midió a través de una escala de 4 puntos que va desde 1 (Nada interesado) a 4 (Muy interesado). En segundo término, el nivel de confianza en el gobierno se evaluó mediante una escala de 4 puntos que varía de 1 (Nada) a 4 (Mucha). Tercero, la confianza interpersonal se calculó con una escala de 4 puntos desde 1 (Casi nunca se puede confiar en la gente) a 4 (Casi siempre se puede confiar en la mayoría de las personas). En cuarto lugar, la ideología política fue medida a través de una escala de autoposicionamiento de 10 puntos en un continuum que va desde 1 (Izquierda) a 10 (Derecha). En quinto término, la responsabilidad moral frente a la política se evaluó con una escala de importancia atribuida por el encuestado al implicarse en cuestiones políticas, se empleó una escala tipo Likert de 5 puntos que va desde 1 (Totalmente en desacuerdo) a 5 (Totalmente de acuerdo). En sexto lugar, la opinión del círculo social percibida fue operacionalizada mediante una escala de 4 puntos que mide el grado de importancia que el encuestado cree que su propio círculo social le atribuye al implicarse en actos de tipo político. Se sitúa en un rango de 5 puntos que va desde 1 (Nada importante) a 5 (Muy importante). Séptimo, la importancia atribuida a la democracia por los participantes se cuantificó en una escala de 10 puntos que va desde 1 (Nada importante) hasta 10 (Muy importante). Octavo, la eficacia política externa se midió a través de un conjunto de 4 ítems asociados con la percepción del encuestado sobre el grado de receptividad del Estado frente a las demandas e intereses de los sectores de la ciudadanía. En contraste, en noveno lugar, la eficacia política interna se evaluó con un conjunto de 4 ítems relacionados con la capacidad autopercibida por el encuestado para participar satisfactoriamente en actos políticos. En ambos tipos de eficacia, se utilizó una escala tipo Likert de 5 puntos que va de 1 (Nada) a 5 (Mucho), con una consistencia interna satisfactoria para la eficacia externa $(\alpha=0,88)$ y la eficacia interna $(\alpha=0,80)$.

Movilización externa: La pertenencia a grupos políticos se midió como una variable dicotómica, en la cual se asigna un valor 1 si el participante forma parte actualmente de alguna organización de carácter político, en tanto el valor 0 refleja la no pertenencia a dichos grupos en la actualidad. El contacto 


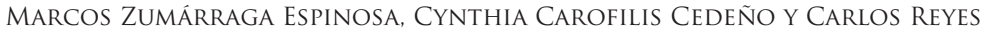 VALENZUELA}

online con organizaciones o agentes políticos que solicitan apoyo, sea para sus propuestas o campañas políticas, fue medido a través de la frecuencia con la que el encuestado ha recibido ese tipo de peticiones. Las opciones de respuesta se ubicaron en un rango de 5 puntos que va desde 1 (Nunca) a 5 (Siempre).

\section{Resultados}

\section{Grado de involucramiento en actividades políticas a través de internet}

La Tabla 2 muestra el porcentaje de los participantes del estudio que se ha involucrado al menos una vez, durante los últimos meses, en cada una de las diferentes actividades políticas que pueden llevarse a cabo en el ámbito online de forma general. Como se puede evidenciar, son aquellas actividades de carácter expresivo y de consumo de información política las que alcanzan mayores tasas participativas en la ciudadanía. Por ejemplo, alrededor del $61 \%$ de la población ha leído noticias de carácter político o relacionadas con las elecciones en los sitios web de los diarios o canales televisivos tradicionales, mientras que cerca del 31\% de la ciudadanía ha discutido con otros sobre política a través de internet.

\section{Tabla 2.}

Porcentaje de participación en actividades de carácter político en la esfera online

\begin{tabular}{l|c} 
& $\begin{array}{c}\text { \% de } \\
\text { Participación }\end{array}$ \\
\hline $\begin{array}{l}\text { Leer noticias de carácter político o relacionadas con las elecciones en los } \\
\text { sitios web de los diarios o canales televisivos tradicionales }\end{array}$ & 60,5 \\
\hline $\begin{array}{l}\text { Visitar páginas web relacionadas con las campañas presidenciales, candidatos } \\
\text { o partidos políticos }\end{array}$ & 43,6 \\
\hline $\begin{array}{l}\text { Ver videos en sitios como Youtube sobre organizaciones políticas, los candi- } \\
\text { datos o campañas presidenciales }\end{array}$ & 42,5 \\
\hline $\begin{array}{l}\text { Leer o publicar comentarios en blogs o páginas web relacionadas con la } \\
\text { política o las campañas electorales }\end{array}$ & 34,4 \\
\hline $\begin{array}{l}\text { Discutir con otros sobre política en internet } \\
\text { políticas, los candidatos o las campañas presidenciales a amigos, familiares, } \\
\text { compañeros de trabajo u otras personas que conozca }\end{array}$ & 29,3 \\
\hline $\begin{array}{l}\text { Intentar persuadir a alguien para que apoye o se oponga a un proyecto políti- } \\
\text { co o candidato a través del internet }\end{array}$ & 19,4 \\
\hline
\end{tabular}




\begin{tabular}{l|c}
\hline $\begin{array}{l}\text { Suscribirse a sitios web para recibir información de carácter político, los } \\
\text { candidatos o las campañas presidenciales }\end{array}$ & 18 \\
\hline $\begin{array}{l}\text { Tomar parte en foros en internet para discutir de asuntos políticos o sociales } \\
\text { relacionados con las elecciones o proyectos políticos }\end{array}$ & 13,2 \\
\hline $\begin{array}{l}\text { Firmar o recolectar firmas para una petición o propuesta a través de internet, } \\
\text { con el objetivo de influir en la política }\end{array}$ & 10,9 \\
\hline $\begin{array}{l}\text { Hacer trabajos, utilizando herramientas y aplicaciones de internet para pro- } \\
\text { mover un candidato, partido político o campaña presidencial }\end{array}$ & 10 \\
\hline Contactar con algún político o funcionario público a través de internet & 8,1 \\
\hline $\begin{array}{l}\text { Escribir un blog sobre temas políticos, los candidatos o las elecciones presi- } \\
\text { denciales }\end{array}$ & 7,5 \\
\hline $\begin{array}{l}\text { Recolectar o contribuir con dinero a organizaciones políticas, candidatos o } \\
\text { campañas presidenciales mediante internet }\end{array}$ & 7,4
\end{tabular}

Fuente: Datos recopilados por el Grupo de Investigaciones Psicosociales (GIPS), Universidad Politécnica Salesiana (UPS), 2017.

Elaborado por: los autores.

En contraste, apenas cerca del $7 \%$ de la población ha recolectado o contribuido dinero a organizaciones políticas, candidatos o las campañas presidenciales mediante internet durante los últimos meses. Esto permite concluir que el activismo político online, en el caso de las últimas elecciones presidenciales, se ha concentrado principalmente en aquellas actividades que requieren un menor gasto de recursos, sean materiales o cognitivos, para su realización. Por otra parte, al enfocar únicamente el uso político de redes sociales, es necesario resaltar que las actividades políticas que pueden efectuarse en dichas plataformas digitales tienden a ser estrictamente de carácter expresivo o de consumo de información política.

La Tabla 3 presenta la tasa de participación de la ciudadanía en el conjunto de actos políticos considerados por el presente estudio para el caso específico de redes sociales. La información se muestra de modo desagregado según se trate de Facebook, Whatsapp o Twitter. Sin importar la red social, recibir contenidos, imágenes, videos y links relacionados con cuestiones políticas, los candidatos o las campañas electorales, constituye la conducta más recurrente en la población durante el último periodo electoral, en la cual $26,2 \%$ de los participantes lo ha hecho a través de Facebook, 25,4\% mediante Whatsapp y 10\% vía Twitter. En contraste, actividades como iniciar o unirse a grupos creados con objetivos de 


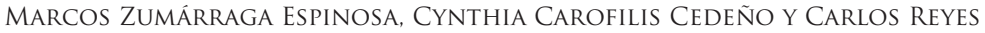 VALENZUELA}

carácter político constituyen prácticas menos frecuentes, con una frecuencia de 9\%, se trate de Facebook, Whatsapp o Twitter.

\section{Tabla 3.}

Porcentaje de involucramiento en actos de tipo político a través de Facebook, Twitter y Whatsapp

\begin{tabular}{l|c|c|c} 
& \multicolumn{3}{|c}{ \% de Participación } \\
\hline & Facebook & Whatsapp & Twitter \\
\hline $\begin{array}{l}\text { Recibir contenidos, imágenes, videos y links rela- } \\
\text { cionados con cuestiones políticas, los candidatos o } \\
\text { las campañas electorales }\end{array}$ & 26,2 & 25,4 & 10 \\
\hline $\begin{array}{l}\text { Poner me gusta o unirse a un grupo iniciado por } \\
\text { una organización política o candidato }\end{array}$ & 18,8 & 11,5 & 7,4 \\
\hline $\begin{array}{l}\text { Compartir imágenes, videos, links y contenidos } \\
\text { relativos a temas políticos, candidatos o campañas } \\
\text { presidenciales }\end{array}$ & 18,8 & 16,4 & 8,2 \\
\hline $\begin{array}{l}\text { Chatear con amigos o conocidos sobre temas políti- } \\
\text { cos, candidatos o campañas electorales }\end{array}$ & 17,6 & 17,4 & 7,1 \\
\hline $\begin{array}{l}\text { Escribir opiniones sobre las elecciones o asuntos } \\
\text { relacionados con la política en su muro o página } \\
\text { personal }\end{array}$ & 12,7 & 11,4 & 6 \\
\hline $\begin{array}{l}\text { Comentar o responder a las opiniones políticas o } \\
\text { relacionadas con las elecciones en las páginas de } \\
\text { otras personas }\end{array}$ & 12,3 & 11,4 & 7 \\
\hline $\begin{array}{l}\text { Unirse a un grupo creado por amigos u otras } \\
\text { personas para tratar temas políticos o apoyar un } \\
\text { candidato o campaña presidencial }\end{array}$ & 8,1 & 4,8 & 4,4 \\
\hline $\begin{array}{l}\text { Iniciar un grupo con el objetivo de promover ideas } \\
\text { políticas, candidatos o campañas presidenciales }\end{array}$ & 6 & 6,1 & 4,6 \\
\hline $\begin{array}{l}\text { ID-UP- Pachakutik } \\
\text { AP }\end{array}$ & 0,35 & - & - \\
\hline AP-PSE & $-0,09$ & 0,1 & 0,16 \\
\hline Pachakutik & - & - & - \\
\hline PSC & - & 0,54 & 0,73 \\
\hline PSE & 0,22 & 0,41 & 0,3 \\
\hline PSP & - & 0,26 & 0,35 \\
\hline $\begin{array}{l}\text { UE } \\
\text { UP }\end{array}$ & & 0,32 \\
\hline
\end{tabular}

Fuente: Datos recopilados por el Grupo de Investigaciones Psicosociales (GIPS), Universidad Politécnica Salesiana (UPS), 2017.

Elaborado por: los autores. 
Decisión de voto atribuida a fuentes de información online

Se informa sobre la relevancia de diferentes fuentes de información disponibles en internet respecto a las decisiones de voto en la ciudadanía. Para ello, se solicitó a las personas participantes del estudio seleccionar aquellos medios sociales o sitios web que, a su juicio, les proporcionaron información importante para decidir por quién votar en la segunda vuelta de las elecciones presidenciales ecuatorianas a celebrarse el 2 abril del 2017.

Los sitios web o medios sociales contemplados en este caso fueron los siguientes: Facebook, Twitter, Whatsapp, blogs de temas políticos o de opinión, Youtube y portales web de la prensa escrita o canales televisivos (véase Gráfico 1). Los resultados indican que los portales web de la prensa escrita y los canales televisivos, así como Facebook han sido las fuentes de información que mayor relevancia han tenido para la toma de decisiones sobre el voto.

El $62 \%$ consideró que los sitios web de la prensa escrita o los canales televisivos les proporcionaron información importante para decidir por quién votar, mientras que cerca del $60 \%$ de la muestra hizo lo propio en relación con la red social Facebook. Por el contrario, Twitter, ha sido el medio social que menor relevancia ha tenido para la población (16\%). Esto puede explicarse por la menor cantidad de usuarios que Twitter registra frente a otras plataformas digitales como Facebook o Whatsapp a nivel global. 


\section{Gráfico 1.}

Fuentes de información online consideradas importantes para la decisión de voto

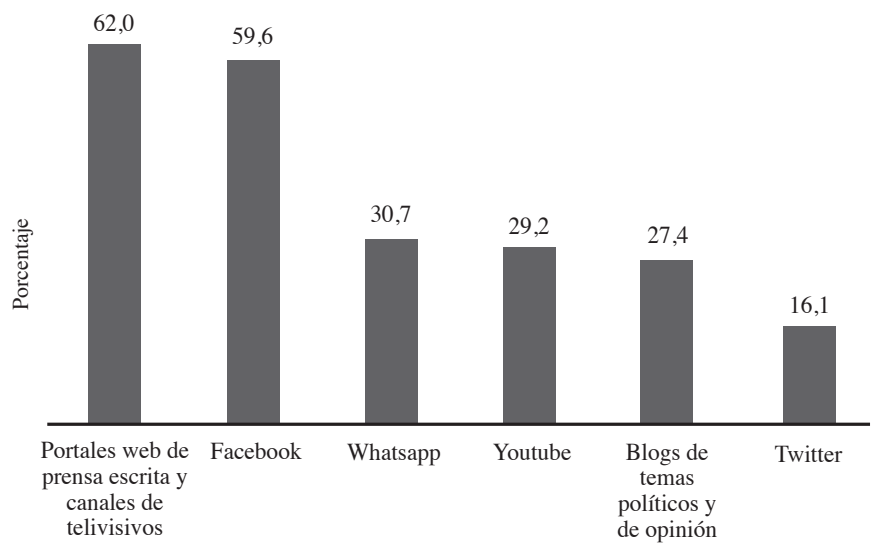

Fuente: Datos recopilados por el Grupo de Investigaciones Psicosociales (GIPS), Universidad Politécnica Salesiana (UPS), 2017.

Elaborado por: los autores.

Destaca el fuerte vínculo que la ciudadanía mantiene con los medios tradicionales como fuentes de información válida que refleja, por un lado, la supremacía de los medios de comunicación masivos en relación con el consumo de información política y, por otra, del aumento considerable de Facebook. En complemento de esto último, salvo para el caso de Twitter, al menos la cuarta parte del electorado ha recurrido a las plataformas digitales como fuentes válidas de información política en estas últimas elecciones, lo que demuestra la creciente importancia que internet va adquiriendo como espacio de influencia y difusión de información política entre la población ecuatoriana.

Efecto de las variables de control y las emociones en la participación política de tendencia online

Para probar el efecto que las variables control y las emociones presentan 
sobre las conductas políticas a través de internet, se elaboraron dos modelos de regresión múltiple por Mínimos Cuadrados Ordinarios (MCO). En el primer modelo, se evalúa el efecto de las respuestas emocionales hacia los candidatos presidenciales sobre la participación política online, en tanto en el segundo modelo, se considera como variable dependiente el uso político de redes sociales. Asimismo, en cada modelo se incluye un extenso conjunto de variables de control que conceptualmente se encuentran relacionadas con el activismo político, sea en sus formas convencionales o a través de internet. La razón de emplear un conjunto tan amplio de variables de control responde al interés de realizar una prueba suficientemente conservadora sobre el rol de las emociones como factor movilizador de la ciudadanía hacia las actividades de carácter político, tal como se ha utilizado en otras investigaciones (Valentino, Brader, Groenendyk, Gregorowicz, \& Hutchings, 2011).

En este caso, se pretende evaluar si se observa un efecto directo de las emociones, tanto en la participación política online como la participación política vía redes sociales, una vez que se ha controlado el impacto del resto de variables que también pueden incidir sobre estas conductas participativas. Como se señaló anteriormente, se evalúan las respuestas emocionales positivas y negativas respecto a los dos candidatos presidenciales de manera separada, lo que permite comparar el efecto movilizador de los estímulos emocionales generados por cada candidato sobre la población.

Los resultados de las regresiones múltiples realizadas se presentan en la Tabla 4. Para facilitar la lectura, la tabla incluye únicamente los coeficientes de regresión no estandarizados (b), los coeficientes de regresión estandarizados (BETA) y sus respectivos valores-p (expresados con asteriscos). Los coeficientes b representan el efecto que cada variable independiente ejerce sobre las respectivas variables dependientes contempladas. En aquellos predictores que posean valores-p que estén por debajo del 5\% se considera que su efecto sobre la variable dependiente es estadísticamente significativo, lo que se traduce en que puede asumirse que dicho efecto existe a nivel poblacional con una probabilidad de estar en lo correcto de al menos $95 \%$. 


\section{MARCoS ZUMÁRRAGA ESPINOSA, CYNTHIA CAROFILIS CEDEÑO Y CARLOS REYES VALENZUELA}

\section{Tabla 4.}

Regresión múltiple de la participación política online y la participación politica a través de redes sociales. Influencia de las emociones considerando variables de control

\begin{tabular}{l|c|c|c|c} 
& \multicolumn{2}{|c|}{$\begin{array}{c}\text { Participación Política } \\
\text { Online }\end{array}$} & \multicolumn{2}{c}{$\begin{array}{c}\text { Participación Política } \\
\text { en Redes Sociales }\end{array}$} \\
\hline Predictores & b & Beta & B & Beta \\
\hline Emociones & & & & \\
\hline Candidato Lenín Moreno & $0,27^{*}$ & 0,07 & $0,66^{* *}$ & 0,11 \\
\hline Respuesta emocional positiva & $0,13^{* * *}$ & 0,15 & $0,20^{* * *}$ & 0,15 \\
\hline Respuesta emocional negativa & & & & \\
\hline Candidato Guillermo Lasso & 0,01 & 0 & 0,31 & 0,05 \\
\hline Respuesta emocional positiva & $-0,02$ & $-0,02$ & $-0,06$ & $-0,04$ \\
\hline Respuesta emocional negativa & & & & \\
\hline Características Sociodemográficas & $-1,12^{*}$ & $-0,06$ & $-0,96$ & $-0,03$ \\
\hline Sexo (Femenino) & $-0,06^{* *}$ & $-0,09$ & $-0,06 *$ & $-0,06$ \\
\hline Edad & & & & \\
\hline Recursos & $-0,29$ & $-0,03$ & $-1,1 * *$ & $-0,07$ \\
\hline Estatus Socioeconómico & & & & \\
\hline Factores digitales & $0,49^{* *}$ & 0,08 & 0,48 & 0,05 \\
\hline Uso de Internet & $0,59 * *$ & 0,07 & $0,82^{* *}$ & 0,07 \\
\hline Eficacia Política de Internet & & & & \\
\hline Motivaciones y actitudes & $2,16^{* * *}$ & 0,22 & $2,38^{* * *}$ & 0,16 \\
\hline Interés en la Política & $-0,38$ & $-0,04$ & $-0,57$ & $-0,04$ \\
\hline Confianza en el Gobierno & 0,47 & 0,04 & $0,87^{*}$ & 0,05 \\
\hline Confianza Interpersonal & $-0,09$ & $-0,02$ & $-0,08$ & $-0,01$ \\
\hline Ideología Política & $0,80^{* *}$ & 0,07 & $1,08^{*}$ & 0,07 \\
\hline $\begin{array}{l}\text { Responsabilidad moral frente a la } \\
\text { política }\end{array}$ & $1,18^{* * *}$ & 0,10 & $1,12^{*}$ & 0,06 \\
\hline Opinión del círculo social percibida & 0,31 & 0,03 & $-0,2$ & $-0,01$ \\
\hline Importancia de la democracia & $0,49^{* * *}$ & 0,14 & $0,70^{* * *}$ & 0,13 \\
\hline Eficacia Política Interna & $0,21^{*}$ & 0,07 & $0,39 * *$ & 0,09 \\
\hline Eficacia Política Externa & $5,02^{* * *}$ & 0,10 & $11,46^{* * *}$ & 0,14 \\
\hline Movilización externa & $1,97^{* * *}$ & 0,18 & $3,96^{* * *}$ & 0,24 \\
\hline Pertenencia a Grupos Políticos & 3,55 & & 5,68 & \\
\hline $\begin{array}{l}\text { Contacto online con organizaciones o } \\
\text { agentes políticos }\end{array}$ & 0,34 & & 0,31 & \\
\hline Constante & 1136 & & 1136 & \\
\hline R2 Ajustado & & & & \\
\hline N & & & & \\
\hline & & & & \\
\hline
\end{tabular}

Nota: ${ }^{*} \mathrm{p}<0,05(5 \%),{ }^{* *} \mathrm{p}<0,01(1 \%), * * * \mathrm{p}<0,001(0,1 \%)$. Los coeficientes Beta hacen referencia a los coeficientes de regresión estandarizados.

Fuente: Datos recopilados por el Grupo de Investigaciones Psicosociales (GIPS), Universidad Politécnica Salesiana (UPS), 2017.

Elaborado por: los autores. 
Por último, los coeficientes BETA son ideales para comparar el tamaño de los efectos generados por los predictores sobre la variable dependiente, siendo recomendable realizar contrastes únicamente en aquellos predictores cuyos efectos sean estadísticamente significativos.

El bloque de características socio-demográficas presenta un efecto estadísticamente significativo recurrente en el caso de la edad, con la particularidad de que la relación tiene un sentido inverso. Este resultado es coherente con las expectativas teóricas, puesto que la participación política a través de internet, en general, constituye un campo de preferencia para la población más joven. El sexo, por otra parte, sugiere diferencias significativas únicamente en torno a la participación política online concebida de modo general, ya que aparece que es mayor en hombres, pero no se encuentran diferencias en el caso de las redes sociales. El estatus socioeconómico como variable de recursos, presenta un efecto diferenciado en la participación política, siendo significativo únicamente en el uso político de redes sociales.

Sin embargo, el signo de los coeficientes de regresión es negativo en los dos modelos de regresión, lo que sugeriría que, al menos en el caso de las últimas elecciones presidenciales, fueron los sectores de menor estatus socioeconómico quienes mostraron una tendencia de mayor involucramiento en actos políticos de carácter online. Respecto a los factores digitales, la frecuencia en el uso de internet solo registró una relación significativa con la participación política online en general, mientras que la eficacia política de internet mantuvo una influencia significativa sobre ambos tipos de participación política.

En relación a las variables motivacionales, el interés en la política, la eficacia política interna y la eficacia política externa muestran efectos significativos positivos sobre ambas variables dependientes, lo cual coincide con los hallazgos que usualmente se reportan en la literatura especializada respecto al tema (Anduiza, Cantijoch, Gallego, \& Salcedo, 2010; Jennings \& Zeitner, 2003; Nam, 2012). Por otro lado, variables como la responsabilidad moral y la opinión del círculo social percibida presentan una relación significativa positiva con cada variable dependiente, aun cuando se controla el efecto del resto de factores explicativos considerados. 
Esto sugiere que concebir la participación política como una responsabilidad moral y que cuando el círculo social propio tiene una opinión favorable sobre la implicación en actos de carácter político, se incrementa la propensión de un individuo hacia el activismo político online. En tanto que la confianza interpersonal solo parece tener una relación estadísticamente significativa con la participación política a través de redes sociales. Esto podría explicarse por el hecho de que las redes sociales están compuestas por una elevada proporción de contactos conocidos cuya opinión, sea positiva o negativa, puede condicionar las actividades que alguien realiza en estas plataformas digitales. En este caso, la confianza en los otros aparece fundamental para facilitar el activismo político en las redes sociales.

Adicionalmente, variables como la confianza en el gobierno, la ideología política y la importancia de la democracia no poseen un efecto significativo sobre la participación política online, sea global o centrada en redes sociales, una vez se controla el efecto del resto de variables explicativas propuestas. Finalmente, de las variables control, la pertenencia a grupos políticos y el contacto vía internet con organizaciones políticas en busca de apoyo para sus propuestas o campañas, muestran efectos positivos significativos sobre las formas de participación política a través de internet. En otras palabras, tanto la pertenencia a grupos políticos como haber recibido la solicitud de participar en actividades políticas, por parte de las organizaciones políticas, hace más probable que una persona asuma un rol activo en el ámbito político online.

En el ámbito de las emociones, se encuentran dos resultados relevantes:

a. Las respuestas emocionales sean positivas o negativas, tienen un efecto movilizador sobre la ciudadanía en términos de activismo político vía internet, aunque las emociones negativas generan un mayor impacto. Considerando los coeficientes estandarizados, es posible comprobar que la respuesta emocional negativa tiene un efecto movilizador que tiende a ubicarse entre las variables con mayor efecto explicativo, tanto en el caso de la participación política online en general, como respecto al uso político de las redes sociales. De este modo, este resultado permite 
confirmar la hipótesis establecida en relación al efecto movilizador de las emociones sobre el comportamiento político de los individuos, esta vez haciendo énfasis en la esfera online. El efecto directo de las emociones sobre la participación política online puede verificarse incluso cuando se realizan controles rigurosos con una amplia gama de variables conceptualmente relacionadas, lo cual coincide con los criterios de confirmación propuestos por Valentino et al. (2011).

b. El candidato Lenín Moreno fue quien generó estímulos emocionales con un efecto político-movilizador sobre el electorado. El respaldo u oposición a su proyecto político generó respuestas emocionales en la ciudadanía que se relacionaron estadísticamente con su nivel de involucramiento en actos políticos a través de internet. Por el contrario, este efecto no pudo verificarse en el caso del candidato Guillermo Lasso, lo que podría explicarse que las respuestas emocionales hacia el candidato Lasso estuvieron determinadas, hasta cierto punto, por las respuestas emocionales respecto al candidato Moreno, dadas las condiciones de control implantadas en los modelos de regresión construidos. En otras palabras, las emociones positivas hacia el candidato Lasso pudieron configurar una respuesta a las emociones negativas generadas respecto al candidato Moreno y viceversa, ya que en ambos casos, se obtienen coeficientes de correlación de Pearson significativos y moderados, $\mathrm{r}>0,60, \mathrm{p}<0,05$. No obstante, dado el carácter transversal de los datos obtenidos, esta relación causal no puede ser verificada ni confirmada de forma consistente.

Los resultados arrojados por las regresiones efectuadas sobre la participación política online, tanto en su forma global como a través de redes sociales, permiten deducir, en cierta medida, las estrategias políticas empleadas por los candidatos presidenciales en este ámbito. Resulta claro que en un momento electoral el activismo político a través de internet está fuertemente conectado con las campañas presidenciales, en la cual un mayor involucramiento en actos políticos online puede traducirse en un mayor respaldo u oposición hacia las campañas políticas de los candidatos. 
En otras palabras, el activismo político online podría asociarse con un debilitamiento o reforzamiento de dichas campañas, lo cual a su vez tendría efectos indirectos sobre los resultados electorales. Los antecedentes políticos y personales del candidato Moreno, la organización política a la que pertenece, así como la influencia de la figura del ex presidente Rafael Correa, pudieron haber dotado de una capacidad político-movilizadora a través de las emociones provocadas en la ciudadanía, fenómeno que no se reprodujo en el caso del candidato Lasso. A modo especulativo, esta diferencia pudo haberse conectado con los resultados electorales que le permitieron al candidato Moreno, finalmente, la victoria presidencial.

\section{Discusión}

El presente estudio presenta interés en dos ámbitos: primero, pone de manifiesto la influencia significativa del entorno online en la participación política de la ciudadanía en la segunda vuelta electoral en Ecuador. En segundo lugar, aporta evidencia empírica a favor del papel movilizador de las emociones en relación a dicho activismo político digital.

Respecto al primer punto, los resultados muestran un porcentaje significativo de actividades políticas desplegadas previamente a la elección, destacándose una participación activa en el ámbito online y más específicamente a través de redes sociales. Precisamente, la función del entorno online posibilita distintos tipos de actividades, que varían desde adquirir información política, participar en debates hasta donar dinero a alguna organización o candidato, las cuales resultan de interés en relación a cómo la ciudadanía participa en tiempos electorales.

Complementario a esto, el entorno online se presenta como una fuente de información consolidada para la decisión del voto y no como una mera alternativa a medios más tradicionales de información. Esto lleva a la consideración sobre qué función están desempeñando las redes sociales y las alternativas online en torno a la participación política y la decisión del voto. Al respecto, los resultados presentados aquí se relacionan con un efecto democratizador del internet y las redes sociales, permitiendo que 
más personas estén informadas, accedan a diversas fuentes de información, propiciando una participación política que no esté limitada por falta de recursos y favorezca condiciones de igualdad entre diversos grupos sociales.

En este marco, cabe resaltar la relación inversa detectada entre el estatus socioeconómico y la participación política online, sea de modo general o en el caso de redes sociales. Esto coincide con hallazgos que afirman que el internet puede funcionar como una "puerta de acceso" para aquellas personas que usualmente se encuentran excluidas del proceso político, gracias a la reducción del costo de participación que posibilita (Kim et al., 2015). Dado esto, el factor motivacional adquiere una relevancia central para explicar las conductas políticas en un entorno menos costoso como lo es el internet.

En relación al segundo punto, se analiza el papel movilizador de las emociones, el cual tiende a intensificarse cuando se trata de la participación política de carácter expresivo o vinculado al consumo de información. Como se pudo constatar en los modelos estadísticos efectuados, el impacto de las respuestas emocionales ya sean positivas o negativas, presenta un incremento cuando la variable explicada pasa a ser el uso político de redes sociales. Esto puede deberse a que el índice general de participación política online incorpora también actividades de mayor alcance y más costosas como la donación de dinero, realizar contactos con funcionarios públicos o escribir un blog político, en tanto las conductas políticas en redes sociales únicamente incluyen actividades centradas en el consumo de información o la expresión de la voz política del ciudadano, actividades que, como se ha señalado, son menos costosas.

Asimismo, es posible afirmar que este efecto movilizador de las emociones se activa momentáneamente, lo que resulta especialmente claro en la participación en redes sociales. Los eventos políticos de alto perfil tales como las elecciones presidenciales, favorecen la creación de comportamientos políticos transitorios en la ciudadanía, impulsados por los esfuerzos de campaña de partidos y agentes políticos (Morris \& Morris, 2013; Valentino et al., 2011). En este punto, las emociones amplificarían temporalmente su efecto político-movilizador durante las elecciones dado un contexto sociopolítico con una carga emocional de alta incertidumbre. 
Los estudios en Psicología Social sugieren que el clima emocional está influenciado por hechos o políticas institucionales que se orientan a generar experiencias emocionales compartidas (De Rivera \& Paéz, 2007). En este sentido, el efecto movilizador transitorio de las emociones observado en la participación en redes sociales tiene relación con un clima emocional igualmente momentáneo presente en los discursos emocionales generados por los candidatos.

Del mismo modo, los resultados son coherentes con la teoría de la Inteligencia Afectiva (Marcus \& MacKuen, 1993), que pronostica el potencial movilizador de las emociones para mejorar la interacción de las personas con el ambiente. De acuerdo a esta teoría, las emociones no son rasgos estables sino dinámicos que permiten reevaluar las posiciones iniciales y ser más flexibles para examinar información novedosa ligada a eventos recientes. En esta investigación, coincidimos con los hallazgos encontrados por Crigler, Just, y Belt (2006) acerca de la relación entre emociones y atención a los medios televisivos, puesto que tanto las emociones positivas como las negativas motivan la participación política, en este caso, buscar información por internet, compartir contenidos online y discutir sobre política con otros.

A pesar de esto, el efecto de las emociones negativas no es constante sino esporádico, ya que se esfumaría una vez que se deja de percibir una situación amenazante (Marcus \& MacKuen, 1993). Esto explicaría que durante la cúspide emocional de la campaña electoral, quienes habitualmente no comparten en sus páginas contenidos políticos lo hagan y se prevea que dejen de hacerlo pasadas las elecciones. Las implicaciones de estos supuestos derivan en que los contextos de alta emocionalidad como las campañas electorales podrían convertirse en escenarios de posibles aprendizajes políticos, que convoquen a la ciudadanía a interesarse, expresarse y participar. Estos resultados, sin embargo, dependen en gran medida de la interrelación que se establece entre medios tradicionales y redes sociales que eligen focalizar determinados temas como asuntos de interés (Salcudean \& Muresan, 2017). Al igual que en los medios tradicionales, las redes sociales pueden ser cooptadas por lógicas mercantiles o élites políticas, que posicionan y 
enmarcan discursos de acuerdo a sus intereses.

Respecto a la intensidad afectiva hacia los candidatos, los datos coinciden con otros estudios realizados en contextos de reelección (Brussino, Alonso, \& Imhoff, 2011) en la cual la intensidad emocional hacia el sistema político resulta determinante en la respuesta emocional hacia los candidatos. Esto, en relación con este estudio, se observa en las respuestas emocionales hacia Lenín Moreno las cuales motivan las reacciones emocionales hacia Guillermo Lasso. Además, esta relación de dependencia sugeriría la posibilidad del "efecto boomerang" (Garramone, Atkin, Pinkleton, \& Cole, 1990) en el cual las campañas negativas hacia un candidato terminan perjudicando a aquel que las utiliza. Siguiendo este argumento, la campaña contra la imagen de Lenín Moreno, serviría para polarizar las actitudes que ya se tenían ante este candidato y ante Guillermo Lasso, con lo cual las reacciones emocionales hacia el primero estarían condicionando las reacciones hacia el segundo.

A partir de estos hallazgos, podemos concluir que el rol de Internet como una "tecnología afectiva" (Serrano-Puche, 2016) proporciona un canal que modela las emociones y participa en la constitución de subjetividad (Serrano-Puche, 2016). Esto podría entenderse con la posibilidad que conforman las redes de democratizar los flujos de información, al generar un escenario de debate y discusión que pueda convocar e incluir a sectores tradicionalmente marginados de la política. En este sentido, el escenario político ecuatoriano de las últimas elecciones se caracteriza por una tendencia de los sectores populares a involucrarse en formas de participación online, como resultado de la interacción de dos factores clave: la reducción de las barreras de acceso provocadas por el internet y los estímulos emocionales generados por el ambiente de campaña electoral y los candidatos presidenciales. Aunque la tendencia detectada puede desvanecerse una vez celebradas las elecciones presidenciales, los resultados del presente estudio reflejan que la esfera online, en efecto, puede establecerse en un espacio que permite disminuir las brechas de participación política que tradicionalmente han persistido entre los más y menos favorecidos. 


\section{Limitaciones y futuros estudios}

La investigación presenta algunas limitaciones a considerar: se trata de un estudio transversal que informa de un momento específico, en este caso, semanas previas a la elección. Sería recomendable efectuar estudios de panel que posibiliten confirmar los resultados presentados aquí, en específico, sobre la frecuencia de uso del entorno online en la participación política y del efecto movilizador de las emociones.

Por otro lado, la elección de la muestra presenta algunas dificultades para promover una representatividad, ya que hay una sobrerrepresentación y está conformada únicamente por habitantes que residen en el DMQ. Al respecto, resulta de interés que futuros estudios indaguen en la participación política en otras ciudades del país, atendiendo a las características demográficas representativas de los electores. Asimismo, este estudio explora la participación política online en un contexto electoral, lo cual plantea la interrogante sobre cómo estará relacionada a formas de participación offline, y qué emociones específicas, positivas y negativas, promueven el efecto movilizador descrito aquí. Los resultados presentados alientan a seguir explorando sobre los aspectos que intervienen en los diversos modos de participación política.

Una segunda limitación refiere a que el impacto de las emociones en los resultados aquí informados puede encontrarse subestimado, debido a elevada cantidad de variables de control incorporadas a los modelos de regresión, que pueden terminar opacando la capacidad explicativa de las respuestas emocionales. No obstante, la introducción de variables de control permite capturar los efectos indirectos que las emociones generarían sobre la participación política online a través de otras potenciales variables moduladoras tales como el interés en la política o variables motivacionales consideradas.

Esto, según Valentino et al. (2011) permite tener mayor seguridad de que las emociones efectivamente influyen de forma directa sobre las conductas políticas, y no a través de otras variables mediadoras. Dada la escasa investigación sobre el rol de las emociones en las conductas 
políticas para el contexto ecuatoriano, se plantea como punto de partida para posteriores investigaciones determinar la presencia de efectos estadísticos directos. En este sentido, resultaría orientativo identificar empíricamente los efectos indirectos de las emociones sobre las conductas políticas online en futuros estudios, para lo cual se pueden emplear técnicas estadísticas como modelos de ecuaciones estructurales.

Otro aspecto a reflexionar sobre la participación política online es considerarla como una dimensión "pasiva" que carece de un impacto en los resultados de un proceso político, lo cual contrasta con acciones de movilización social. Al respecto, el entorno digital puede considerarse como un campo de ensayo para personas que usualmente están alejadas de la esfera política, facilitando su puesta en contacto con ésta y una futura implicación en actividades políticas en el mundo real. Esto permitiría conjeturar un efecto movilizador de las conductas políticas digitales (incluyendo las redes sociales) sobre otras formas de activismo político.

Con respecto al efecto democratizador del internet encontrado en este trabajo, es necesario resaltar que pudo observarse únicamente en el caso del activismo politico a través de redes sociales. Esto implica que el internet facilita el acceso fundamentalmente a aquellas formas de participacion de carácter expresivo o de consumo de información. Por el contrario, en la esfera real dicho efecto actuaría solo de forma parcial, puesto que si bien es cierto, el internet estimula las diversas motivaciones haciendo que aquellos que se encontraban desconectados de la política adquieran interés por vincularse a ésta; los costos del activismo político real seguirán limitando a aquellos que no cuenten con los recursos necesarios. Esta cuestión debería examinarse en próximos estudios para el contexto ecuatoriano, y evaluarlas en distintos momentos políticos a los informados aquí.

En síntesis, el internet tiene el potencial para generar ciudadanos mejor informados y más interesados en la política, no obstante, configura una herramienta con límites y posibilidades que no democratiza en sí misma, sino más bien está expresando los discursos políticos que describen cómo una sociedad se apropia de la tecnología (Papacharissi, 2009). De este modo, los discursos respecto al potencial de internet, la idoneidad de la información 
en redes sociales o la necesidad de regular la información que surge en estos espacios digitales, evidencian a su vez los diferentes discursos sobre cómo está ocurriendo dicha apropiación tecnológica.

Al respecto, surgen las siguientes preguntas, entre otras: ¿La participación política online posibilita la generación de debates más provechosos y más alternativas de libertad de expresión que en ausencia de tal participación?, ¿La participación online facilita la inclusión de personas y/o favorece la conversión en audiencias más críticas?, ¿El interés en esta participación online representa algo efímero y transitorio o promueve una participación más estable en el tiempo? o ¿La participación online conlleva a acciones offline? Estas y otras interrogantes ponen de manifiesto que la participación online y las emociones expresadas en tales ámbitos, conforman áreas de creciente complejidad que requieren ser consideradas por distintas instituciones y disciplinas académicas relacionadas a esta temática. 


\section{Referencias}

Anduiza, E., Cantijoch, M., Gallego, A., \& Salcedo, J. (2010). Internet y participación política en España. Opiniones y Actitudes.

Bekafigo, M. A., \& Mcbride, A. (2013). Who Tweets About Politics? Social Science Computer Review, (July), 1-19. http://doi. org/10.1177/0894439313490405

Bosnjak, M., Galesic, M., \& Klicek, B. (2007). "Determinants of Online Political Participation in Croatia". Drustvena Istrazivanja, 17(4-5), 747-769.

Brussino, S., Alonso, D., \& Imhoff, D. (2011). "Dimensiones culturales, afectivas y cognitivas del comportamiento de voto al kirchnerismo". Psicologia \& Sociedade, 27(2), 351-361.

Campaña electoral se anticipa en las redes. (2016, agosto 14). El Universo. Guayaquil. Recuperado a partir de http:/www.eluniverso.com/ noticias/2016/08/14/nota/5741244/campana-electoral-se-anticipa-redes

Carvajal, A. M. (2017, enero 2). "Las redes sociales también son "tarima” de los presidenciables". El Comercio. Quito.

Corporación Latinobarómetro. (2016). Informe 2016. Buenos Aires. Recuperado a partir de www.latinobarometro.org

Crigler, A., Just, M., \& Belt, T. (2006). "The three faces of negative campaigning: the democratic implications of attack ads, cynical news, and fear-arousing messages". En D. P. Redlawsk (Ed.), Feeling politics (pp. 135-163). New York.

De Marco, S., \& Robles Morales, J. M. (2012). "Uso de los blogs políticos: análisis de algunos factores determinantes”. Arbor, 188(756), 689-705. 


\section{MARCoS ZUMÁRraGa ESPINOSA, CYNTHIA CAROFILIS CEDEÑO Y CARLOS REYES VALENZUELA}

http://doi.org/10.3989/arbor.2012.756n4005

De Rivera, J., \& Paéz, D. (2007). "Emotional climate, human security, and cultures of peace". Journal of Social Issues, 63(2), 233-253. http://doi. org/10.1111/j.1540-4560.2007.00506.x

Espinar-Ruiz, E., \& González-Río, M. J. (2015). “Uso de Internet y prácticas políticas de los jóvenes españoles". Convergencia, 22(69), 13-38.

Garramone, G., Atkin, C., Pinkleton, B., \& Cole, R. (1990). "Effects of Negative Political Advertising on the Political Process". Journal of Broadcasting \& Electronic Media, 34(3), 299-311. http://doi. org/10.1080/08838159009386744

Gibson, R., \& Cantijoch, M. (2013). "Conceptualizing and Measuring Participation in the Age of the Internet: Is Online Political Engagement Really Different to Offline?". The Journal of Politics, 75(3), 701-716. http://doi.org/10.1017/S0022381613000431

Gibson, R. K., Lusoli, W., \& Ward, S. J. (2005). "Online participation in the UK: Testing a "contextualised" model of Internet effects". British Journal of Politics and International Relations, 7(4), 561-583. http:// doi.org/10.1111/j.1467-856X.2005.00209.x

Gil de Zúñiga, H., Molyneux, L., \& Zheng, P. (2014). “Social media, political expression, and political participation: Panel analysis of lagged and concurrent relationships". Journal of Communication, 64(4), 612-634. http://doi.org/10.1111/jcom.12103

Gil de Zúñiga, H., Veenstra, A., Vraga, E., \& Shah, D. (2010). "Digital Democracy: Reimagining Pathways to Political Participation". Journal of Information Technology \& Politics, 7(1), 36-51. http://doi. org/10.1080/19331680903316742 
Instituto Nacional de Estadísticas y Censos. (2016). Tecnologías de la Información y Comunicaciones ( TIC'S ) 2016.

Jennings, M. K., \& Zeitner, V. (2003). "Internet use and civic engagement: A longitudinal analysis”. Public Opinion Quarterly, 67(3), 311-334.

Jiang, L. (2016). "The Effects of the Internet on Online and Offline Political Participation among Citizens in Australia". En 66th Annual International Conference of British Political Science Association.

Kim, Y., Ph, D., Russo, S., Ph, D., Amnå, E., \& Ph, D. (2015). “The Longitudinal Relation Between Online and Offline Political Participation Among Youth at Two Different Developmental Stages". APSA Annual Meeting | September 3-6, 2015 | San Francisco , CA, 36(2002), 2015. http://doi.org/10.1177/1461444813518391

Lehman, K., Verba, S., Brady, H. E., Schlozman, K. L., Verba, S., \& Brady, H. E. (2010). "Weapon of the Strong ? Participatory Inequality and the Internet". Perspectives on Politics, 8(2), 487-509. http://doi. org/10.1017/S1537592710001210

Marcus, G., \& MacKuen, M. (1993). “Anxiety, Enthusiasm, and the Vote: The Emotional Underpinnings of Learning and Involvement During Presidential Campaigns". American Political Science Review, 87(3), 672-685. http://doi.org/10.2307/2938743

Marcus, G., Neuman, R., \& Mackuenn, M. (2007). “Inteligencia afectiva y juicio político”. Sociológica, 22(63), 253-266.

Milošević-Đorđević, J. S., \& Žeželj, I. L. (2016). "Civic activism online: Making young people dormant or more active in real life? Computers in Human Behavior, 70. http://doi.org/10.1016/j.chb.2016.12.070

Morris, D. S., \& Morris, J. S. (2013). Digital inequality and participation 


\section{MARCoS ZUMÁRraGa ESPINOSA, CYNTHIA CAROFILIS CEDEÑO Y CARLOS REYES VALENZUELA}

in the political process: Real or imagined? Social Science Computer Review, 31(5), 589-600. http://doi.org/10.1177/0894439313489259

Nam, T. (2012). Dual effects of the internet on political activism: Reinforcing and mobilizing. Government Information Quarterly, 29(SUPPL. 1), S90-S97. http://doi.org/10.1016/j.giq.2011.08.010

Papacharissi, Z. (2009). Handbook of Internet Politics. Handbook of Internet Politics, 1-35. http://doi.org/10.1111/1478-9302.12016_66

Perea, E. A., Cantijoch, M., Gallego, A., Salcedo, J., Anduiza Perea, E., \& Sociológicas, C. de I. (2010). Internet y participación política en España. Opiniones y Actitudes.

Pimentel Junior, J. T. P. (2010). Razão e emoção: o voto na eleição presidencial de 2006. Opinião Pública, 16(2), 516-541. http://doi.org/10.1590/ S0104-62762010000200009

Punín, M. I., Martínez, A. C., \& Rencoret, N. A. (2014). Medios digitales en Ecuador: perspectivas de futuro. Digital Media in Ecuador - Future Perspectives. Revista Comunicar, 21, 199-207. http://doi.org/10.3916/ C42-2014-20

Redlawsk, D. P. (2006). Feeling Politics: Emotion in Political Information Processing. (D. P. Redlawsk, Ed.) (Vol. 53). New York: Palgrave Macmillan. http://doi.org/10.1057/9781403983114

Rojas, C. (2017). Carlos Andrés Vera habla sobre la política y las redes sociales | El Comercio. El Comercio. Recuperado a partir de http://www. elcomercio.com/actualidad/politica/carlos-andres-vera-habla-politica. html

Salcudean, M., \& Muresan, R. (2017). El impacto emocional de los medios tradicionales y los nuevos medios en acontecimientos sociales. 
Comunicar, 50(XXV), 109-118. http://doi.org/https://doi.org/10.3916/ C50-2017-10 |

Serrano-Puche, J. (2016). Internet y emociones: nuevas tendencias en un campo de investigación emergentes. 01-2016, 46, 19-26. Recuperado a partir de http://www.revistacomunicar.com/index. php? contenido $=$ detalles \&numero $=46 \&$ articulo $=46-2016-02$

Sheppard, J. (2015). Measuring online and offline participation: problems and solutions from the Australian case. En European Survey Research Association annual conference (pp. 1-19). http://doi.org/10.1017/ CBO9781107415324.004

Shulman, S. (2009). The Case Against Mass E-mails: Perverse Incentives and Low Quality Public Participation in U.S. Federal Rulemaking. Policy \& Internet, 1(1), 23-53. http://doi.org/10.2202/1948-4682.1010

Vaccari, C., Valeriani, A., Barberá, P., Bonneau, R., Jost, J. T., Nagler, J., \& Tucker, J. A. (2015). Political expression and action on social media: Exploring the relationship between lower- and higher-threshold political activities among twitter users in Italy. Journal of Computer-Mediated Communication, 20(2), 221-239. http://doi.org/10.1111/jcc4.12108

Valentino, N. A., Brader, T., Groenendyk, E. W., Gregorowicz, K., \& Hutchings, V. L. (2011). Election Night's Alright for Fighting: The Role of Emotions in Political Participation. The Journal of Politics, 73(1), 156-170. http://doi.org/10.1017/S0022381610000939

Valenzuela, S., Park, N., \& Kee, K. F. (2009). Is There social capital in a social network site?: Facebook use and college student's life satisfaction, trust, and participation1. Journal of Computer-Mediated Communication, 14(4), 875-901. http://doi.org/10.1111/j.1083-6101.2009.01474.x

Verba, S., Schlozman, K. L., Lehman, K., \& Brady, H. E. (1995). Voice and 61 
Equality. Civic voluntarism in American Politics. London: Harvard University Press.

Yang, H. C., \& Dehart, J. L. (2016). Social Media Use and Online Political Participation Among College Students During the US Election 2012. Social Media + Society, 1-18. http://doi.org/10.1177/2056305115623802

Zamora, R., \& Losada, J. C. (2011). Candidates Political Image : Towards an Integration Between its Rational and Emotional Dimension Hacia una integración de su dimensión racional y emocional. Cuadernos de Información, (29), 9-24.

Zhang, W., Johnson, T. J., Trent Seltzer, \& Bichard, S. L. (2009). The Revolution Will be Networked, The Influence of Social Networking Sites on Political Attitudes and Behavior. Social Science Computer Review, (June 2014), 1-18. http://doi.org/10.1177/0894439309335162 